Review

\title{
Plasmodium falciparum Thioredoxin Reductase (PfTrxR) and Its Role as a Target for New Antimalarial Discovery
}

\section{Sara E. McCarty ${ }^{1,2, \dagger}$, Amanda Schellenberger ${ }^{2,3, \dagger}$, Douglas C. Goodwin ${ }^{4}$, Ngolui Rene Fuanta ${ }^{4}$,} Babu L. Tekwani ${ }^{5}$ and Angela I. Calderón ${ }^{2, *}$

1 College of Sciences and Mathematics, Auburn University, Auburn, AL 36849, USA;

E-Mail: sem0026@auburn.edu

2 Department of Drug Discovery and Development, Harrison School of Pharmacy, Auburn University, Auburn, AL 36849, USA; E-Mail: ans0038@auburn.edu

3 College of Agriculture, Auburn University, Auburn, AL 36849, USA

4 Department of Chemistry and Biochemistry, Auburn University, Auburn, AL 36849, USA; E-Mails: goodwdc@auburn.edu (D.C.G.); rfn0001@tigermail.auburn.edu (N.R.F.)

5 National Center for Natural Products Research and Department of BioMolecular Sciences, School of Pharmacy, University of Mississippi, Oxford, MS 38677, USA;

E-Mail: btekwani@olemiss.edu

$\dagger$ These authors contributed equally to this work.

* Author to whom correspondence should be addressed; E-Mail: aic0001@auburn.edu; Tel.: +1-334-844-8333; Fax: +1-334-844-8331.

Academic Editor: Derek J. McPhee

Received: 12 May 2015 / Accepted: 17 June 2015 / Published: 22 June 2015

Abstract: The growing resistance to current antimalarial drugs is a major concern for global public health. The pressing need for new antimalarials has led to an increase in research focused on the Plasmodium parasites that cause human malaria. Thioredoxin reductase (TrxR), an enzyme needed to maintain redox equilibrium in Plasmodium species, is a promising target for new antimalarials. This review paper provides an overview of the structure and function of TrxR, discusses similarities and differences between the thioredoxin reductases (TrxRs) of different Plasmodium species and the human forms of the enzyme, gives an overview of modeling Plasmodium infections in animals, and suggests the role of Trx functions in antimalarial drug resistance. TrxR of Plasmodium falciparum is a central focus of this paper since it is the only Plasmodium TrxR that has been crystallized and P. falciparum is the species that causes most malaria cases. It is anticipated that the 
information summarized here will give insight and stimulate new directions in which research might be most beneficial.

Keywords: thioredoxin reductase; Plasmodium falciparum; malaria; animal models

\section{Introduction}

Malaria, a deadly disease characterized by high fevers and chills, is caused by Plasmodium spp. protozoan parasites that are transmitted to humans via a bite from an infected Anopheles mosquito [1]. An estimated 198 million cases of malaria occurred worldwide in 2013 with approximately 584,000 deaths. Four parasite species are known to cause malaria in humans, $P$. falciparum, $P$. vivax, $P$. malariae, and $P$. ovale, while a fifth species that mainly infects monkeys, $P$. knowlesi, has recently been found in human cases of malaria as well [2]. Most malaria cases and deaths are caused by P. falciparum, and P. falciparum and $P$. vivax pose the greatest challenges to public health [3]. $P$. ovale and $P$. vivax in humans and $P$. cynomolgi in monkeys can cause recurrent malaria due to their ability to persist in the liver of hosts in a dormant hypnozoite form [4,5]. Plasmodium species have three stages in their life cycle: the pre-erythrocytic cycle, which occurs in the vector and then the human liver, the erythrocytic cycle, which occurs in human red blood cells, and the sporogonic cycle, which occurs mainly in the insect vector [6]. The erythrocytic stage is primarily responsible for pathogenesis of malaria and is most harmful to the host [7].

$P$. falciparum relies on two antioxidant systems to relieve oxidative stress and maintain redox equilibrium within the parasite [8]. These are the glutathione and thioredoxin systems. The thioredoxin system consists of thioredoxin reductase (TrxR), its substrate, thioredoxin (Trx), and NADPH. TrxR contains a dithiol/disulfide active site and serves as a cellular protein disulfide reductase, while Trx is an electron donor for a multitude of enzymes, including ribonucleotide reductases, thioredoxin peroxidases and methionine sulfoxide reductases [9].

Recently, reviews have been written covering Plasmodium's antioxidant systems describing in detail how TrxR works as known at that time [10], comparing antioxidant systems of parasites, including $P$. falciparum, in order to further understanding of parasite biology, parasite-host interactions, and drug resistance [11], reviewing the current knowledge on thioredoxin superfamily and its role in Plasmodium [12], and using the thioredoxin system or TrxR as a plausible drug target [13-21].

This review presents an overview of PfTrxR structure and functions, especially comparison with TrxR identified in different rodent and primate Plasmodium spp., and potential role of TrxR in antimalarial drug resistance. Additionally, we have identified the areas and potential gaps in the knowledge related to Plasmodium TrxR. This would stimulate further research on potential of TrxR as target for antimalarials against Plasmodium species that are infectious to humans vis-à-vis design and development of more specific inhibitors of $P f$ TrxR as potential new antimalarial drug leads. 


\section{Key Aspects of PfTrxR Active Sites for Enzyme Inhibition}

The structure of PfTrxR complexed with its substrate, thioredoxin (Figure 1), has been solved only recently [22,23]. Although PfTrxR shows distinct characteristics in comparison to TrxR identified in other Apicomplexan parasites and the human counterparts (e.g., the extended insertion loop addressed below), there isa striking similarity between the TrxR from different Plasmodium species. As with all known TrxR enzymes, PfTrxR is a homodimeric protein where each subunit divides into three major domains. The FAD-binding domain roughly includes residues 38 to 197 and 321 to 390 . The residues between the two sequence components of the FAD-binding domain (i.e., 198-320) have been assigned as the NADPH-binding domain, and the C-terminal segment of the subunit from about S391 to the C-terminus (G541) is referred to as the interface domain. The homodimeric structure of PfTrxR contains two fully functional pairs of redox centers. The N-terminal redox center is referred to as such because the bulk of residues for FAD and NADPH binding as well as electron transfer associated with this center are provided by the N-terminal domains (38-320). However, it is important to point out that two residues provided by the C-terminal interface domain of the second subunit (H509 and E514) contribute to the function of this center. The location of the $\mathrm{N}$-terminal dual-cysteine redox center is immediately adjacent to the isoalloxazine ring system of the FAD prosthetic group. The so-called C-terminal redox center is accounted for almost entirely by the C-terminal nine amino acids (G533-G541), most notably cysteines 535 and 540. A notable insertion loop also interacts with both the substrate and the enzyme. The flexible conformation of the C-terminal arm and the extended insertion loop set PfTrxR apart from human TrxR and contribute to differences in substrate binding and reduction [22]. Formation of the enzyme-substrate (PfTrxR-Trx) complex results in rearrangement of the C-terminal active site and the insertion loop, which is a necessary conformational change for the enzyme's catalytic cycle [23]. This feature of $P f \operatorname{TrxR}$ is similar to that of human TrxR.

\subsection{N-Terminal Redox Center}

Four amino acids in the N-terminal domain are essential to TrxR activity: Cys 88, Cys 93, His 509, and Glu 514. Cys 88 and Cys 93 are buried in the protein and are the endpoints of a strictly conserved CVNVGC sequence that forms a single five-amino-acid helical turn [24,25]. This helical turn dramatically distorts what is otherwise a typical alpha helix, and is a typical feature of disulfide reductases. In the enzyme's oxidized state, Cys 88 and Cys 93 are joined to one another through a disulfide bond. The disulfide resides directly above $\mathrm{N} 1$ of the flavin isoalloxazine ring system. [23,25]. Replacement of either Cys 88 or Cys 93 with either Ser or Ala results in the complete loss of TrxR activity in reducing DTNB. Cys 93 is implied to be the thiolate flavin charge transfer thiol for the N-terminal redox center after testing conditions stated previously [25]. His 509 and Glu 514 are known to be essential to the active site and are contributed by a strongly conserved region of the interface domain of the second subunit. The $\varepsilon 2$ nitrogen of the histidine imidazole is in closest proximity to the sulfur of Cys 88 . Simultaneously, the $\delta 1$ nitrogen of the His 509 imidazole is in H-bonded contact with the Glu 514 carboxylate [23]. Thus, it is proposed that these two residues form a catalytic dyad where the general acid/base properties of His 509 are modulated by Glu 514 [25]. In support of this idea, substitution of His 509 with either Gln or Ala decreases the PfTrxR $k_{\text {cat }}$ for NADPH-dependent DTNB reduction by two orders of magnitude with 
relatively small impact on the apparent $K_{\mathrm{M}}$ values for either substrate [25]. Similarly, substitution of Glu 514 with Ala produces an order of magnitude decrease in activity [25]. In any case, residual albeit minimal activity in H509A variants suggest that another residue may be able to substitute for His 509 to a limited extent in catalysis $[25,26]$.

\subsection{C-Terminal Redox Center}

The C-terminal redox center is found on the highly conformationally flexible C-terminus of the interface domain. In contrast to mammalian TrxR C-terminal redox center, which is composed of

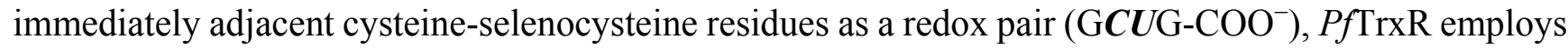
a two-cysteine redox pair (Cys 535 and Cys 540) where the Cys residues are separated by an intervening four-amino-acid linker (GCGGGKCG-COO${ }^{-}$) [22]. Several amino acids and a disulfide ring contribute to the active site of the C-terminal redox center. Cys 535 is hydrogen bonded to neighboring amino acids on the flexible arm of the terminal domain. Cys 535 has several important functions including electron transfer, reduction of thioredoxin and serving as the resolving cysteine of the intermolecular disulfide. Cys 540 is also involved in electron transfer and reduction of the substrate, but it has also been suggested to be the most physiologically relevant nucleophilic cysteine of the C-terminus. This is due to its flexibility, since it is located on the flexible arm of the $\mathrm{C}$ terminus, and has been determined to be the ideal nucleophile to approach the substrate [23]. The C-terminal amino acid (Gly 541) affects enzymatic activity by forming a salt bridge with Lys58 with its backbone carboxylate as well as a hydrogen bond between its amide nitrogen and the carbonyl oxygen of Gly 534 [24]. The hydrogen bond is more important in stabilizing the $\beta$-turn- $\beta$ motif and serves as an anchor for the reduction of the $\mathrm{C}$-terminal domain disulfide. The $\mathrm{C}$-terminal redox center has a twenty-member disulfide ring when $\mathrm{C}$-terminal Cys residues 535 and 540 are oxidized, similar to the N-terminal extension, which has an impact on activity. Deletion of Lys 539 from between the $\mathrm{C}$-terminus cysteines in the ring, leaving it with only seventeen atoms, has a significant reduction on the enzyme activity. Therefore, C-terminal domain chain length is important to the ring's functionality [24].

\subsection{Enzyme Catalytic Cycle}

TrxR-catalyzed reduction of Trx by NADPH is proposed to require a priming stage where NADPH binding is followed by formation a $\mathrm{FADH}^{-}$to $\mathrm{NADP}^{+}$charge transfer complex. Along with NADP ${ }^{+}$ release, $\mathrm{FADH}^{-}$is reoxidized at the expense of Cys 93, forming Cys- $\mathrm{S}^{-}$to $\mathrm{FAD}$ charge transfer complex and a thiol for subsequent disulfide exchange at Cys 88. This form of TrxR is proposed to be the start and end of the enzyme's catalytic cycle. Through intersubunit dithiol-disulfide exchange with the disulfide $\mathrm{C}$-terminal redox center, the $\mathrm{N}$-terminal redox center is returned to its fully oxidized state (i.e., FAD, Cys 88-Cys 93 disulfide) and the C-terminal redox center is reduced from its disulfide to dithiol state. In a sequence essentially identical to the priming steps, NADPH binding produces the $\mathrm{NADP}^{+}$to $\mathrm{FADH}^{-}$charge transfer complex which again transitions to a Cys 93 thiolate to FAD charge transfer complex and a Cys 88 thiol. With both the $\mathrm{N}$ - and $\mathrm{C}$-terminal redox centers fully reduced, Trx reacts with the C-terminal redox center through dithiol: disulfide exchange to convert the Trx disulfide substrate to the corresponding reduced dithiol Trx product. In so doing, the C-terminal redox center returns to its oxidized disulfide state, and the next reaction cycle can begin [27]. 


\subsection{Interactive Cavity and Interface}

Within PfTrxR there are a narrow active site cavity and a monomer-monomer interface. In the cavity, Tyr 101 and His 104 alter the stereochemical properties of the cavity in PfTrxR compared to the Gln and Leu in cavity in human thioredoxin reductase (hTrxR). The narrowness is suggested to be due to a H-bond with Asp 112 on the $\alpha 3$ helix from the other monomer. Due to the narrowness of the cavity, it is difficult for larger molecules to access the PfTrxR active site [22,23]. Indeed, it has been suggested that the cavity can host smaller, more amphipathic molecules [22]. The PfTrxR interface is a bent $\alpha 3$ helix because of the unique presence of Met 105 and Phe 109. It contributes to dimer stability and to substrate binding of thioredoxin due to the bulky Met 105 and Phe 109 inducing a bend that goes on to stimulate a conformational change downstream on the same interface. PfTrxR assumes an anti-parallel conformation that allows H-bonding particularly between Asp 121 and Asn 122 [22,23].

\subsection{Insertion Loop}

PfTrxR has an insertion loop of amino acids, His 438-Ser 456, in a cleft within the protein. This loop consists of nineteen residues in PfTrxR, but only five residues in hTrxR. Different parts of the loop interact with different substrates or intermolecular residues. Some interact only with residues of $P f \operatorname{TrxR}$, others with the C-terminus in particular, and three interact with reductase and substrate interface residues. Deletion of residues 438-452 of the loop produced a no change in the $K_{\mathrm{M}}$ for the pseudosubstrate DTNB but a seven fold increase in $K_{\mathrm{M}}$ for the true substrate, Trx. Interestingly, the $k_{\text {cat }}$ value with respect to both substrates were only moderately diminished. These data suggest that the insertion loop is important for interaction and complex formation between PfTrxR and its PfTrx substrate. His 438 may be particularly important in reduction of the disulfide bond. The H438A PfTrxR variant can account almost entirely for the decrease in $k_{\text {cat }}$ observed for the loop deletion variant, but itself shows no impact on the $K_{\mathrm{M}}$ with respect to PfTrx [23].

$P f$ TrxR has a higher $K_{\mathrm{M}}$ and lower $k_{\text {cat }}$ for both $P f$ Trx and DTNB than is observed for hTrxR [23,27,28]. Replacing Cys with Sec in the second position of the redox center, which makes PfTrxR more like hTrxR, shows activities with E. coli Trx lower than that of the wild-type PfTrxR. This result may be due to incomplete ligation of the peptide containing Sec during incorporation into the enzyme using a semisynthetic technique. Why P. falciparum did not evolve to use Sec is still unknown [24].

\section{TrxR: Human and Plasmodium Species}

\subsection{TrxR Isoenzymes}

Mammalian TrxR can be found as three isoenzymes: TrxR1, TrxR2, and TGR. hTrxR1 is the cytosolic form of the enzyme while hTrxR2 is the miotochondrial form. Both hTrxR1 and hTrxR2 have the CVNVGC active site and the FAD-binding domain and NADPH-binding domain common to Plasmodium TrxR forms. However, hTrxR2 has a 33-amino acid extension at the N-terminus that is thought to be a mitochondrial translocation signal. A third form, TGR, sometimes referred to as hTrxR3, exists mainly in developing sperm. It is more similar to hTrxR1 than to hTrxR2 and, unlike other TrxRs, has a N-terminal glutaredoxin domain, hence the name "thioredoxin glutathione reductase," or TGR [18]. 
Like hTrxR, PfTrxR has an isoform found in the cytosol (PfTrxR1) and one found in the mitochondria (PfTrxR2) [29].

\subsection{Identities in the Sequences of Human and Plasmodium Species TrxRs}

The predicted amino acid sequences of TrxR from different Plasmodium spp were obtained from GenBank. Pairwise and multiple sequence alignments were performed with ClustalW and the shaded box plots were generated using BoxShade. PfTrxR shows a 40\% sequence identity to the longer hTrxR2 and a $42 \%$ identity to the shorter hTrxR 1 . It has a $77 \%-80 \%$ identity to the five other listed Plasmodium species (Table 1). The TrxR in P. berghei has been localized to the cytosol [30]. This could explain its slightly greater identity to hTrxR1 than hTrxR2. As described in Figure 2 the CVNVGC active site is conserved for all six species of Plasmodium and human isoenzymes. Conversely, the GCGGGKC C-terminal is conserved in all six Plasmodium species but is not present in the human isoenzymes. Sequences of TrxR for P. falciparum (P. falc.), P. vivax (P. viv), P. yoelli yoelli (P. yoel.), P. berghei ANKA (P. berg), H. sapiens 1 (H. sap 1), H. sapiens 2 (H. sap2), P. cynomolgi strain B (P. cyn), and P. knowlesi strain $\mathrm{H}$ (P. know) were obtained from the National Center for Biotechnology Information (NCBI) protein databank.

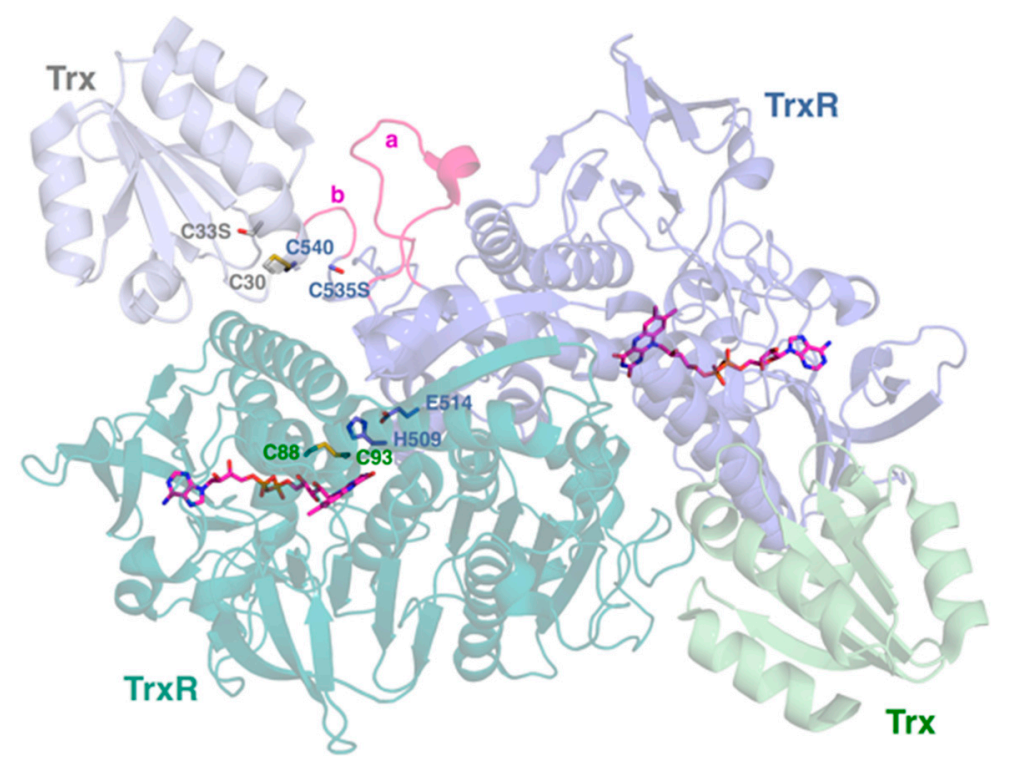

Figure 1. The dimeric structure of $P$. falciparum thioredoxin reductase (TrxR). The two subunits of TrxR are shown with their bound FAD cofactors in magenta. The redox active disulfide (C88-C93) of the N-terminal redox center is shown for the TrxR monomer on the lower left. H509 and E514 that modulate the reactivity of this N-terminal redox center are supplied by the TrxR monomer on the upper right. Each monomer is bound to substrate Trx via intermolecular disulfide (TrxR C540 to Trx C30). The positions of C535 (TrxR) and C33 (Trx) are indicated by serine residues. These substitutions were made in order to trap the intermolecular disulfide between TrxR and Trx [23]. The 535 and 540 residues shown are supplied by the TrxR monomer on the upper right. The Plasmodium-unique insertions H438-S452 and G536-K539 are highlighted by $a$ and $b$, respectively. Coordinates are drawn from PDB accession 4J56 [23]. The figure was generated using PyMOL 1.6.0.0. 
Table 1. Amino acid sequence identities between Plasmodium sp. TrxRs the human cytosolic and mitochondrial isoforms.

\begin{tabular}{|c|c|c|c|c|c|c|c|c|}
\hline & H. sapiens (hTrxR1) & H. sapiens (hTrxR2) & P. falciparum & P. vivax & P. yoelii yoelii & P. berghei ANKA & P. knowlesi Strain H & $\begin{array}{c}\text { P. cynomolgi } \\
\text { Strain B } \\
\end{array}$ \\
\hline Accession Number & AAB35418 & AAD19597 & CAA60574 & EDL45043 & EAA21839 & XP_679935 & XP_002258509 & XP_004221759 \\
\hline Number of amino acids & 497 & 524 & 541 & 546 & 638 & 542 & 623 & 628 \\
\hline Identity to P. falciparum (\%) & 42 & 40 & 100 & 77 & 79 & 79 & 79 & 80 \\
\hline Identity to H. sapiens (hTrxR2) (\%) & 54 & 100 & 40 & 41 & 41 & 40 & 41 & 41 \\
\hline Identity to H. sapiens (hTrxR1) (\%) & 100 & 54 & 42 & 41 & 42 & 42 & 41 & 41 \\
\hline
\end{tabular}

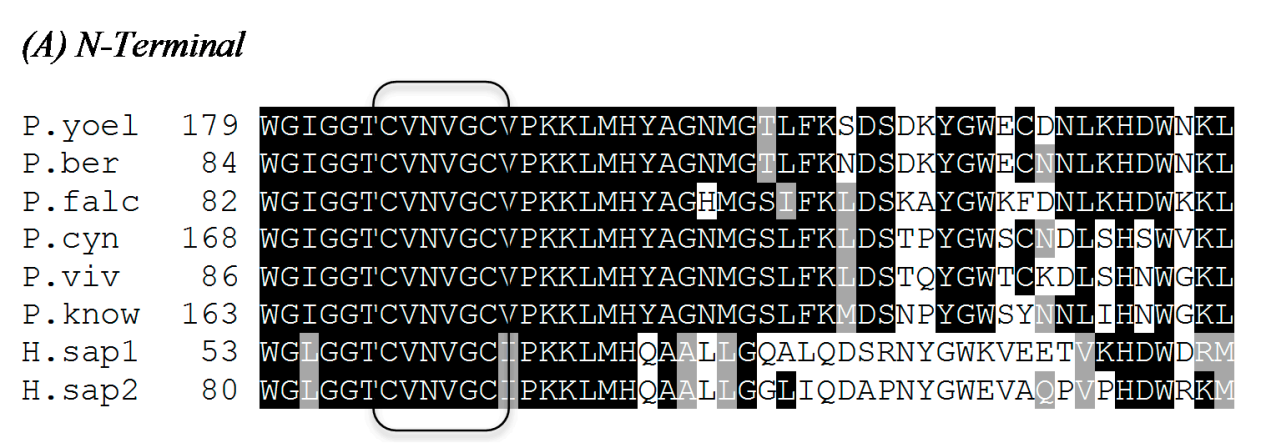

\section{(B) C-Terminal}

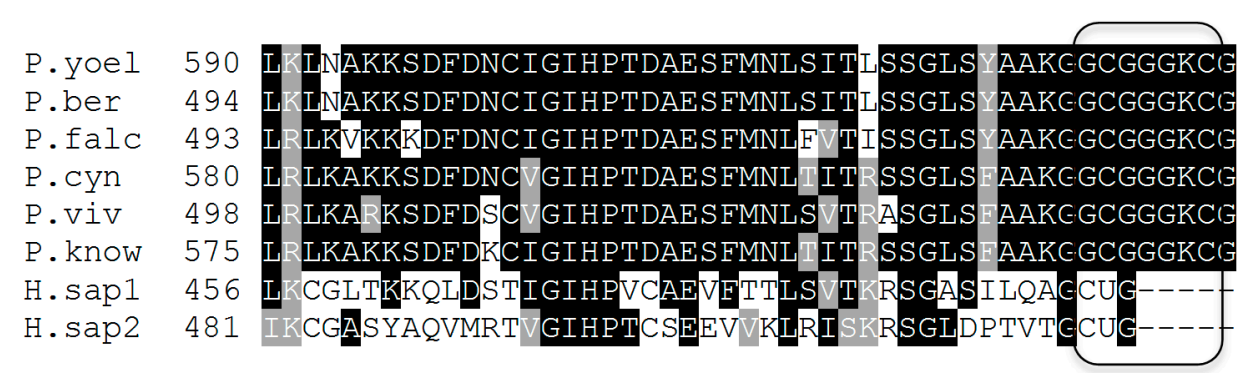

Figure 2. Active site comparison between human and Plasmodium species for (A) the N-Terminal and (B) the C-Terminal. Black shaded amino acids represent identity with the corresponding black shaded amino acids of other species' TrxR while grey represents amino acids with similar properties and no shading represent no identity. The figure was generated with ClustalW2 MSA (Multiple Sequence Alignment) program. 


\subsection{Correlation of Enzymatic Inhibitory Activity between PfTrxR and the TrxRs of Other} Plasmodium Species

\subsubsection{Selective Inhibitory Activity toward PfTrxR versus hTrxR}

Winkler, et al. created aculeatin analogues with Michael acceptor inhibitors and tested their effects on PfTrxR activity. Michael acceptor groups have been described as capable of covalently binding to TrxR. Consistent with a mechanism of covalent modification for TrxR inactivation, all of these analogs, including (-)-aculeatin A required pre-incubation with TrxR before any inhibitory effect could be detected. Assays based on Trx reduction by a couple of aculeatin analogs consistently showed greater extents of inhibition than those recorded using DTNB but not for (-)-aculeatin. The mentioned aculeatin analogs were able to reduce the activity of the C-terminal active site with only marginal effect on the FAD active site. This mechanistic behavior suggested the involvement of other plasmodial target apart from the classical Michael acceptors. These data suggest that the C-terminal redox center is the target for TrxR modification by (-)-aculeatin [31].

Andricopulo, et al. have discovered that, despite of high reactivity of hTrxR at the selenocysteine active site, there are compounds that can selectively inhibit PfTrxR. Thus, an inhibitor of PfTrxR is a plausible antimalarial in humans because the inhibitor is less reactive towards hTrxR. The selective inhibitor of PfTrxR is believed to interact with other structures or amino acid residues of the enzyme, such as the intersubunit region (interactive cavity), as opposed to the conserved C-terminal redox center [21].

\subsubsection{Selective Inhibitory Activity toward PfTrxR versus Other Plasmodium Species TrxRs}

Theobald, et al. have generated a toolbox of novel, recombinant reagents and assays to explore inhibition of TrxR as the potential mechanism of action of one of the subsets of compounds recently identified through phenotypic screening, the Tres Cantos antimalarial set (TCAMS), which comprises 13533 molecules that are highly potent growth inhibitors of the P. falciparum [32].

Seven new inhibitors of TrxR against $P$. falciparum, $P$. vivax, $P$. berghei, and $P$. yoelli isoforms were identified. These data seem to indicate that little to no selectivity between Plasmodium species exists. When these inhibitory compounds were tested with respect to TrxR of the four Plasmodium species, they were found to show noncompetitive inhibition. It was confirmed that PfTrxR shows a ping-pong mechanism with its substrate and it is hypothesized that these inhibitors could prevent the dithiol-disulfide exchange in the C-terminal domain of the subunit, which would prevent the solvent exposure needed to reduce Trx [32]. All these inhibitors showed $<20 \%$ cytotoxicity at $10 \mu \mathrm{M}$ using human cell cytotoxicity data generated with the human line cell HepG2.

Assessment of the specificity of these new inhibitors remains unclear given the absence of studies of the atomic-resolution structure of Plasmodium species TrxR either alone or in complex with one or more of them. These studies will help to rationalize the findings in the next level of testing in animal models. This will be particularly challenging when it has been suggested that the rodent malaria parasite $P$. berghei does not depend on TrxR in its murine host [33]. The lack of essentiality of $P$. berghei TrxR in rodent models should be closely observed when testing the inhibitors due to high redundancies and multiple back-ups in the redox system that most likely ensure the survival upon loss of individual components [34]. 
So far, the bottleneck in finding new druggable inhibitors of PfTrxR is the presence of suicide substrates, a subgroup of mechanism-based inhibitors among the most of the active compounds found in literature [35]. Rational drug design approaches are needed to overcome this obstacle of mechanism-based suicide inhibitors for instance a sort of prodrugs needed to be activated by the C-terminal arm before blocking the FAD active site and hence the entire electron transfer chain.

\subsection{Essentiality of TrxR in Plasmodium falciparum and Other Plasmodium Species}

Since $P$. falciparum lacks glutathione peroxidase and catalase, the thioredoxin systems are essential for maintaining redox homeostasis and antioxidant defense [8]. PfTrxR's substrates, PfTrx1 in its cytosol and $P f \operatorname{Trx} 2$ in its mitochondria, are necessary electron donors in these systems [21]. Oxidative stress is higher than usual in $P f$ erythrocytes due to ingestion of host cell hemoglobin and the subsequent release of free heme. This leads generation to superoxide anions that are further reduced to $\mathrm{H}_{2} \mathrm{O}_{2}$ by superoxide dismutase, and $P f$ TrxR systems must then reduce $\mathrm{H}_{2} \mathrm{O}_{2}$ to $\mathrm{H}_{2} \mathrm{O}$ to complete the antioxidant defense. The $P$. falciparum peroxiredoxins, particularly 2-Cys peroxiredoxin Pf Trx-Px1 reduces hydrogen peroxide. $P f$ Trx is responsible for reducing the peroxiredoxin back to the active form and then PfTrxR must in turn reduce Trx back to the active form [36]. In a study conducted by Kranjski, et al., blood stage forms of $P$. falciparum were genetically modified by inserting a TrxR2 knockout construct via transfection. Attempts to generate viable $P$. falciparum parasites in the erythrocytic stages with the TrxR2 null mutants were unsuccessful, indicating that TrxR2 knockout has a lethal affect for P. falciparum [37]. TrxR knockout of $P$. berghei was prepared by Buchholz, et al. The blast analysis showed that the PfTrxR is homologous to TrxR 2, same is true with TrxR from $P$. vivax and $P$. berghei. Mice were infected with $P$. berghei in which $P b \operatorname{Trx}$ had been replaced with a null mutant, pTrxRRep. Results indicate that $P b T r x R$ is essential for optimal growth and viability, but viable parasites were still obtained when PbTrxR was not present [33]. Interestingly, these two results are not in agreement. The differing conditions of the two experiments could contribute to the results; the PbTrxR knockout parasites were tested in vivo using murine models while the PfTrxR knockout was not tested in vivo. The host's antioxidant functions under in vivo conditions and/or differences between antioxidant functions of mouse and human erythrocytes may also contribute to these differences.

\subsection{Animal Models Available to Test PfTrxR Inhibitors}

Murine models, or mice models, are the most commonly used first step in in vivo malaria drug testing, despite the apparent limitations of truly reflecting a malaria infection within a human [38]. Mice are nonprimates that are normally unable to be infected by the Plasmodium species that affect humans. In one mouse model by Badell, et al. the (bg/bg xid/xid nu/nu) BXN mice were immunocompromised in order to sustain $P$. falciparum-parasitized human erythrocytes in vivo. The group experimented using different methods to best suppress the immune response of the mouse to sustain AB human red blood cells and parasitemia. One method was sub-lethal irradiation with cyclophosphamide or injecting di-chloromethylene biphosphonate-(CI, MBP)-encapsulated liposomes which target tissue macrophages and anti-PMN monoclonal antibodies in the mouse. A combination of the two resulted in parasitemia of $3 \%$, but values fluctuated greatly as the immune response still reacted despite measures to suppress or the mouse died [39]. This presents that $P$. falciparum can be modeled promisingly in mice and, therefore, vaccines 
and antimalarials can be tested. Murine models could potentially also be used to model $P$. vivax $[38,40]$. Another model of $P$. falciparum parasitemia in BXN mice was developed by Moreno, et al., who used di-chloromethylene diphosphonate (C12MDP) and NIMP-R14 monoclonal antibody to reduce tissue macrophages and blood leukocytes in the mice to sustain parasitemia for several weeks, with rare deaths occurring. Human AB or A+ blood types that were infected with P. falciparum African NF54 strain and the Thailand T24 strain were engrafted into the mice, whose peripheral blood then became $85 \%-99 \%$ human red blood cells. While parasitemia percentages were still relatively low and some fluctuation observed, maintenance of infection was sustained for up to two months. The model showed a possibility of accepting multiple isolates, and could be used to determine drug susceptibility of a human parasite, pharmacokinetics, and toxicology [41]. Validation of the usefulness of murine models has been established through the identification of antimalarials currently in clinical use. Identification of mefloquine [42], halofantrine [43], and artemisinin derivatives as antimalarials [29,43,44] was done through use of rodent models, indicating that there is correlation and ability to predict the response to treatments by infected humans. An example of the method(s) used to infect and study in vivo P. berghei, P. yoelii, and/or P. chabaudi infection in vivo in a murine model was established by Helmby, et al. [45]. In-vivo P. berghei infections in mice do not have to be immunocompromised because these species of Plasmodium naturally infect mice and, depending on type of experiment, ICR (outbred stock) mice, CD-1 mice, or National Medical Research Institute (NMRI) mice can be used [10,45]. A P. berghei mouse malaria models for evaluation of test compounds for parasitemia suppression, cure or improvement of survival of infected mice has also been described [46].

\section{Primate Models}

Use of a primate model after a murine model confirms the results of drug and/or vaccine efficacy in rodents and is a better prediction of any effects on humans. The owl monkey, Aotus trivirgatus, and Saimiri primate models are capable of sustaining $P$. falciparum and $P$. vivax infections. These species are well characterized and provide a more clear relationship between human infection and antimalarial response than in murine models, making the use of primate models the stage before clinical trials [40,45]. $P$. vivax can be modeled using $P$. cynomolgi infection in rhesus monkeys due to parallel symptoms. Infecting monkeys with a Plasmodium species can be done through the method outlined by Collins [45]. P. cynomolgi is a more useful model species for studying $P$. vivax than $P$. knowlesi since 217 genes are exclusive to $P$. cynomolgi and $P$. vivax while only 17 genes are exclusive to P. knowlesi and P. vivax [4]. P. cynomolgi is the only surrogate model available for testing the compound for antirelapse radical curative activity against hyponozoites [47]. Choice of animal model depends upon cost per test, rate of testing needed, reproducibility of efficacy of the antimalarial, the known sensitivity of the drug, and other factors relevant to the research.

\subsection{PfTrxR and Antimalarial Drug Resistance}

$P$. falciparum has acquired resistance against almost all antimalarial drugs presently under clinical use [48]. Several reports have shown significant role of glutathione (GSH) and associated functions in drug resistance of P. falciparum. A recent report has shown an increased transport of GSH into the parasite digestive vacuoles in P. falciparum strains with a mutated chloroquine resistant transport, which 
confers the CQ resistance [49]. The multidrug resistance-associated protein (PfMRP) was reported to be involved in efflux of GSH; which might contribute to parasite responses to multiple antimalarial drugs [50]. The inhibitors of TrxR have been shown to be active against susceptible as well drug resistant malaria parasites [21]. A direct role of Trx and TrxR in antimalarial drug resistance has not been investigated. In view of a close connection between GSH and Trx pathways and the prominent role of GSH in antimalarial drug resistance; the role of Trx pathway in antimalarial drug resistance may be investigated. A recent study showed that overexpression and nuclear translocation of thioredoxin-1 (Trx-1) are closely associated with hypoxia related drug resistance in $\mathrm{HeG} 2$ cells through the regulation of the metabolism by the oxidative stress response to glycolysis [51]. A curcumin analog sensitized the cisplatin-resistant A549 cells to cisplatin by inhibiting TrxR [52]. Both GSH and Trx metabolism have widely implicated in resistance of cancer cells to chemotherapy [53]. TrxR has been shown to be involved in activation of 5-nitroimidazole and linked to 5-nitroimidazole resistance in Giardia lamblia [54]. Overexpression of TrxR has also been linked to Trx functions that modulate drug-specific cytotoxic responses [55].

\section{Future Studies}

The overview on PfTrxR and other malaria species presented above clearly underline the necessity of Trx related functions for survival of the $P$. falciparum within the host erythrocytes. The malarial Trx system functions in tandem to the glutathione system. Further testing on whether or not viable parasites of P. falciparum and P. berghei could be created under similar TrxR knockout conditions is needed to provide a more definitive answer as to whether or not TrxR is essential for the survival of the human and rodent species. Further TrxR knockout testing with other Plasmodium strains would also be useful in understanding the essentiality of TrxR across Plasmodium species. The crystal structure has been determined only for P. falciparum; therefore, crystallization of the TrxRs of other Plasmodium species could provide information about structural and active site similarities between Plasmodium species and the human form. Also, the crystal structure of $P f$ TrxR may be used as a template to determine structures of TrxR from other malaria species by homology modeling. Striking similarities and sequence homologies between TrxR form different malaria species is important and indicate that the rodent malaria model would be suitable testing the inhibitors of $P f$ TrxR with potent antimalarial activity in vitro in phenotypic $P$. falciparum screening.

\section{Acknowledgments}

AIC is indebted to Harrison School of Pharmacy for the financial support through New Faculty Start up. SEM is grateful for the National Scholars Presidential Scholarship provided by Auburn University for her undergraduate degree. BLT is partially supported by the USDA-ARS under scientific cooperative scientific agreement no. 58-6408-2-0009 with the National Center for Natural Products Research, University of Mississippi. 


\section{Author Contributions}

Conducted the literature search and composed Sections 1, 3.1, 3.2, 3.3.2, 3.4, and 4: S.E.M. Conducted the literature search and composed Sections 2, 3.3.1, and 3.5: A.S. Conducted the literature search and composed Section 3.6: B.L.T. Assisted in the analysis of amino acid sequences similarities among Plasmodium TrxR: N.R.F. Conceived the idea, developed the structure of the paper and supervised the complete preparation: A.I.C. Critical revision of manuscript: S.E.M., A.S., D.C.G., B.L.T., A.I.C. Reviewed and approved the final manuscript: S.E.M., A.S., D.C.G., N.R.F., B.L.T., A.I.C.

\section{Conflicts of Interest}

The authors declare no conflict of interest.

\section{References}

1. Malaria. Available online: http://www.cdc.gov/malaria/about/faqs.html (accessed on 16 February 2015).

2. Malaria. Available online: http://www.who.int/mediacentre/factsheets/fs094/en/ (accessed on 2 February 2015).

3. World Malaria Report 2014. Available online: http://apps.who.int/iris/bitstream/10665/144852/2/ 9789241564830_eng.pdf?ua=1 (accessed on 24 February 2015).

4. Tachibana, S.; Sullivan, S.A.; Kawai, S.; Nakamura, S.; Kim, H.R.; Goto, N.; Arisue, N.; Palacpac, N.M.; Honma, H.; Yagi, M.; et al. Plasmodium cynomolgi genome sequences provide insight into Plasmodium vivax and the monkey malaria clade. Nat. Genet. 2012, 44, 1051-1055.

5. Treatment of Malaria: Guideline for Clinicians (United States). Available online: http://www.cdc.gov/malaria/diagnosis_treatment/clinicians2.html (accessed on 3 March 2015).

6. Life Cycle. Available online: http://www.bio.davidson.edu/people/sosarafova/Assets/Bio307/ ruturakhia/page01.html (accessed on 24 February 2015).

7. Crutcher, J.M.; Hoffman, S.L. Medical Microbiology, 4th ed.; University of Texas Medical Branch: Galveston, TX, USA, 1996; Chapter 83. Available online: http://www.ncbi.nlm.nih.gov/books/ NBK8584/ (accessed on 5 May 2015).

8. Jortzik, E.; Becker, K. Thioredoxin and glutathione systems in Plasmodium falciparum. Int. J. Med. Microbiol. 2012, 302, 187-194.

9. Arnér, E.S.J.; Holmgren, A. Physiological functions of thioredoxin and thioredoxin reductase. Eur. J. Biochem. 2000, 267, 6102-6109.

10. Lakshminarayana, S.; Freymond, C.; Fischli, C.; Yu, J.; Weber, S.; Goh, A.; Yeung, B.K.S.; Ho, P.C.; Dartois, V.; Diagana, T.T.; et al. Pharmacokinetic-pharmacodynamic analysis of spiroindolone analogs and KAE609 in a murine malaria model. Antimicrob. Agents Chemother. 2015, 59, 1200-1210.

11. Müller, S.; Gilberger, T.; Krnajski, Z.; Lüersen, K.; Meierjohann, S.; Walter, R. Thioredoxin and glutathione system of malaria parasite Plasmodium falciparum. Protoplasma 2001, 217, 43-49.

12. Rahlfs, S.; Schirmer, R.; Becker, K. The thioredoxin system of Plasmodium falciparum and other parasites. Cell. Mol. Life Sci. 2002, 59, 1024-1041. 
13. Rahlfs, S.; Nickel, C.; Deponte, M.; Schirmer, R.; Becker, K. Plasmodium falciparum thioredoxins and glutaredoxins as central players in redox metabolism. Redox Rep. 2003, 8, 246-250.

14. Mohring, F.; Pretzel, J.; Jortzik, E.; Becker, K. The redox systems of Plasmodium falciparum and Plasmodium vivax: Comparison, in silico analyses and inhibitor studies. Curr. Med. Chem. 2014, 15, 1728-1756.

15. Jaeger, T.; Flohé, L. The thiol-based redox networks of pathogens: Unexploited targets in the search for new drugs. BioFactors 2006, 27, 109-120.

16. Williams, C., Jr.; Arscott, D.; Müller, S.; Lennon, B.; Ludwig, M.; Veine, D.; Becker, K.; Schirmer, R. Thioredoxin reductase two modes of catalysis have evolved. Eur. J. Biochem. 2000, 267, 6110-6117.

17. Becker, K.; Gromer, S.; Schirmer, R.; Müller, S. Thioredoxin reductase as a pathophysiological factor and drug target. Eur. J. Biochem. 2000, 267, 6118-6125.

18. Müller, S.; Liebau, E.; Walter, R.; Krauth-Siegel, R. Thiol-based redox metabolism of protozoan parasites. Trends Parasitol. 2003, 19, 320-328.

19. Munigunti, R.; Gathiaka, S.; Acevedo, O.; Sahu, R.; Tekwani, B.; Calderon, A. Determination of antiplasmodial activity and binding affinity of curcumin and demethoxycurcumin towards PfTrxR. Nat. Prod. Res. 2014, 28, 359-364.

20. Munigunti, R.; Becker, K.; Brun, R.; Calderon, A. Determination of antiplasmodial activity and binding affinity of selected natural products towards PfTrxR and PfGR. Nat. Prod. Commun. 2013, 8, 1135-1136.

21. Andricopulo, A.; Akoachere, M.; Krogh, R.; Nickel, C.; McLeish, M.; Kenyon, G.; Arscott, L.; Williams, C.; Davioud-Charvet, E. Specific inhibitors of Plasmodium falciparum thioredoxin reductase as potential antimalarial agents. Bioorg. Med. Chem. Lett. 2006, 16, 2283-2292.

22. Boumis, G.; Giardina, G.; Angelucci, F.; Bellelli, A.; Brunori, M.; Dimastrogiovanni, D.; Saccoccia, F.; Miele, A.E. Crystal Structure of Plasmodium falciparum thioredoxin reductase, a validated drug target. Biochem. Biophys. Res. Commun. 2012, 425, 806-811.

23. Fritz-Wolf, K.; Jortzik, E.; Stumpt, M.; Preuss, J.; Iozef, R.; Rahlfs, S.; Becker, K. Crystal structure of the Plasmodium falciparum thioredoxin reductase-thioredoxin complex. Mol. Biol. 2013, 425, 3446-3460.

24. Snider, G.; Dustin, C.; Ruggles, E.; Hondal, R. A mechanistic investigation of the c-terminal redox motif of thioredoxin reductase from Plasmodium falciparum. Biochemistry 2014, 53, 601-609.

25. Gilberger, T.; Walter, R.; Muller, Sylke. Identification and characterization of the functional amino acids at the active site of the large thioredoxin reductase from Plasmodium falciparum. J. Biol. Chem. 1997, 272, 29584-29589.

26. Muller, S. Thioredoxin reductase and glutathione synthesis in Plasmodium falciparum. Redox Rep. 2003, 8, 251-255.

27. McMillan, P.; Arscott, L.; Ballou, D.; Becker, K.; Williams, C., Jr.; Müller, S. Identification of acid-base catalytic residues of high-Mr thioredoxin reductase from Plasmodium falciparum. J. Biol. Chem. 2006, 281, 32967-32977.

28. Bozdech, Z.; Ginsburg, H. Antioxidant defense in Plasmodium falciparum-Data mining of the transcriptome. Malar. J. 2004, 3, 23.

29. Miranda-Vizuete, A.; Damdimopoulos, A.; Pedrajas, J.R.; Gustafsson, J.; Spyrou, G. Human mitochondrial thioredoxin reductase. Eur. J. Biochem. 1999, 261, 405-412. 
30. Su, D.; Novoselov, S.V.; Sun, Q.A.; Moustafa, M.E.; Zhou, Y.; Oko, R.; Hatfield, D.L.; Gladyshev, V.N. Mammalian selenoprotein thioredoxin-glutathione reductase. Roles in disulfide bond formation and sperm maturation. J. Biol. Chem. 2005, 280, 26491-26498.

31. Winkler, M.; Maynadier, M.; Wein, S.; Lespinasse, M.; Boumis, G.; Miele, A.; Vial, H.; Wong, Y. Uncovering new structural insights for antimalarial activity from cost-effective aculeatin-like derivatives. Org. Biomol. Chem. 2015, 13, 2064-2077.

32. Theobald, A.J.; Caballero, I.; Coma, I.; Colmenarejo, G.; Cid, C.; Gamo, F.; Hibbs, M.J.; Bass, A.L.; Thomas, D.A. Discovery and biochemical characterization of Plasmodium thioredoxin reductase inhibitor from an antimalarial set. Biochemistry 2012, 51, 4764-4771.

33. Buchholz, K.; Putrianti, E.D.; Rahlfs, S.; Schirmer, R.H.; Becker, K.; Matuschewski, K. Molecular genetics evidence for the in vivo roles of the two major NADPH-dependent disulfide reductases in the malaria parasite. J. Biol. Chem. 2010, 285, 37388-37395.

34. Patzewitz, E.M.; Wong, E.H.; Muller, S. Dissecting the role of glutathione biosynthesis in Plasmodium falciparum. Mol. Microbiol. 2012, 83, 304-318.

35. Saccoccia, F.; Angelucci, F.; Boumis, G.; Carotti, D.; Desiato, G.; Miele, A.E.; Bellelli, A. Thioredoxin reductase and its inhibitors. Curr. Protein Pept. Sci. 2014, 15, 621-646.

36. Boucher, I.W.; McMillan, P.J.; Gabrielsen, M.; Akerman, S.E.; Brannigan, J.A.; Schnick, C.; Brzozowski, A.M.; Wilkinson, A.J.; Muller, S. Structural and biochemical characterization of a mitochondrial peroxiredoxin from Plasmodium falciparum. Mol. Microbiol. 2006, 61, 948-959.

37. Krnajski, Z.; Gilberger, T.; Walter, R.D.; Cowman, A.F.; Muller, S. Thioredoxin reductase is essential for the survival of Plasmodium falciparum erythrocytic stages. J. Biol. Chem. 2002, 277, 25970-25975.

38. Kaira, B.; Chawla, S.; Gupta, P.; Valecha, N. Screening of antimalarial drugs: An overview. Indian J. Pharmacol. 2006, 38, 7-11.

39. Badell, E.; Pasquetto, V.; Van Rooijen, N.; Druilhe, P. A mouse model for human malaria erythrocytic stages. Parasitol. Today 1995, 11, 235-237.

40. Fidock, D.; Rosenthal, P.; Croft, S.; Brun, R.; Nwaka, S. Antimalarial drug discovery: Efficacy models for compound screening. Nat. Rev. Drug Discov. 2004, 3, 509-520.

41. Moreno, A.; Badell, E.; Van Rooijen, N.; Druilhe, P. Human malaria n immunocompromised mice: New in vivo model for chemotherapy studies. Antimicrob. Agents Chemother. 2001, 45, 1847-1853.

42. Peters, W.; Howells, R.E.; Portus, J.; Robinson, B.L.; Thomas, S.; Warhurst, D.C. The chemotherapy of rodent malaria. XXVI. Studies on mefloquine (WR 142,490). Ann. Trop. Med. Parasitol. 1977, 71, 407-441.

43. Peters, W.; Robinson, B.L.; Ellis, D.S. The chemotherapy of rodent malaria. XLII. Halofantrine and halofantrine resistance. Ann. Trop. Med. Parasitol. 1987, 81, 639-646.

44. Vennerstrom, J.L.; Dong, Y.; Andersen, S.L.; Ager, A.L., Jr.; Fu, H.; Miller, R.E.; Wesche, D.L.; Kyle, D.E.; Gerena, L.; Walters, S.M.; et al. Synthesis and antimalarial activity of sixteen dispiro-1,2,4,5-tetraoxanes: Alkyl-substituted 7,8,15,16-tetraoxadispiro[5.2.5.2] hexadecanes. J. Med. Chem. 2000, 43, 2753-2758.

45. Moll, K.; Ljungström, I.; Perlmann, H.; Scherf, A.; Wahlgren, M. Methods in Malaria Research, 5th ed.; Malaria Research and Reference Reagent Resource Center: Manassas, VA, USA, 2008; pp. 141-152. 
46. Sahu, R.; Walker, L.A.; Tekwani, B.L. In vitro and in vivo anti-malarial activity of tigecycline, a glycylcycline antibiotic, in combination with chloroquine. Malar. J. 2014, 13, 414.

47. Deye, G.A.; Gettayacamin, M.; Hansukjariya, P.; Im-erbsin, R.; Sattabongkot, J.; Rothstein, Y.; Macareo, L.; Fracisco, S.; Bennett, K.; Magill, A.J.; et al. Use of a rhesus Plasmodium cynomolgi model to screen for anti-hypnozoite activity of pharmaceutical substances. Am. J. Trop. Med. Hyg. 2012, 86, 931-935.

48. Sinha, S.; Medhi, B.; Sehgal, R. Challenges of drug-resistant malaria. Parasite 2014, 21, 61.

49. Patzewitz, E.M.; Salcedo-Sora, J.E.; Wong, E.H.; Sethia, S.; Stocks, P.A.; Maughan, S.C.; Murray, J.A.; Krishna, S.; Bray, P.G.; Ward, S.A.; et al. Glutathione transport: A new role for PfCRT in chloroquine resistance. Antioxid Redox Signal. 2013, 19, 683-695.

50. Raj, D.K.; Mu, J.; Jiang, H.; Kabat, J.; Singh, S.; Sullivan, M.; Fay, M.P.; McCutchan, T.F.; $\mathrm{Su}$, X.Z. Disruption of a Plasmodium falciparum multidrug resistance-associated protein (PfMRP) alters its fitness and transport of antimalarial drugs and glutathione. J. Biol. Chem. 2009, 284, $7687-7696$.

51. Zhao, L.; Li, W.; Zhou, Y.; Zhang, Y.; Huang, S.; Xu, X.; Li, Z.; Guo, Q. The overexpression and nuclear translocation of Trx-1 during hypoxia confers on HepG2 cells resistance to DDP, and GL-V9 reverses the resistance by suppressing the Trx-1/Ref-1 axis. Free Radic. Biol. Med. 2015, 82, $29-41$.

52. Zhou, B.; Huang, J.; Zuo, Y.; Li, B.; Guo, Q.; Cui, B.; Shao, W.; Du, J.; Bu, X. 2a, a novel curcumin analog, sensitizes cisplatin-resistant A549 cells to cisplatin by inhibiting thioredoxin reductase concomitant oxidative stress damage. Eur. J. Pharmacol. 2013, 707, 130-139.

53. Sobhakumari, A.; Love-Homan, L.; Fletcher, E.V.; Martin, S.M.; Parsons, A.D.; Spitz, D.R.; Knudson, C.M.; Simons, A.L. Susceptibility of human head and neck cancer cells to combined inhibition of glutathione and thioredoxin metabolism. PLOS ONE 2012, 7, e48175.

54. Leitsch, D.; Burgess, A.G.; Dunn, L.A.; Krauer, K.G.; Tan, K.; Duchêne, M.; Upcroft, P.; Eckmann, L.; Upcroft, J.A. Pyruvate:ferredoxin oxidoreductase and thioredoxin reductase are involved in 5-nitroimidazole activation while flavin metabolism is linked to 5-nitroimidazole resistance in Giardia lamblia. J. Antimicrob. Chemother. 2011, 66, 1756-1765.

55. Eriksson, S.E.; Prast-Nielsen, S.; Flaberg, E.; Szekely, L.; Arnér, E.S. High levels of thioredoxin reductase 1 modulate drug-specific cytotoxic efficacy. Free Radic. Biol. Med. 2009, 47, 1661-1671.

Sample Availability: Not available.

(C) 2015 by the authors; licensee MDPI, Basel, Switzerland. This article is an open access article distributed under the terms and conditions of the Creative Commons Attribution license (http://creativecommons.org/licenses/by/4.0/). 